Article

\title{
Classification of Human Daily Activities Using Ensemble Methods Based on Smartphone Inertial Sensors
}

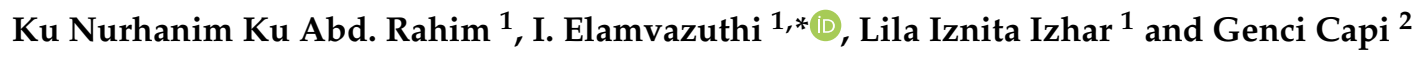 \\ 1 Smart Assistive and Rehabilitative Technology (SMART) Research Group, \\ Department of Electrical and Electronic Engineering, Universiti Teknologi PETRONAS, \\ 32610 Bandar Seri Iskandar, Malaysia; ku_g03269@utp.edu.my (K.N.K.A.R.); lila.izhar@utp.edu.my (L.I.I.) \\ 2 Assistive Robotics Laboratory, Department of Mechanical Engineering, Faculty of Science and Engineering, \\ HOSEI University, Tokyo 184-8584, Japan; capi@hosei.ac.jp \\ * Correspondence: irraivan_elamvazuthi@utp.edu.my; Tel.: +605-3687882
}

Received: 14 September 2018; Accepted: 19 November 2018; Published: 26 November 2018

check for updates

\begin{abstract}
Increasing interest in analyzing human gait using various wearable sensors, which is known as Human Activity Recognition (HAR), can be found in recent research. Sensors such as accelerometers and gyroscopes are widely used in HAR. Recently, high interest has been shown in the use of wearable sensors in numerous applications such as rehabilitation, computer games, animation, filmmaking, and biomechanics. In this paper, classification of human daily activities using Ensemble Methods based on data acquired from smartphone inertial sensors involving about 30 subjects with six different activities is discussed. The six daily activities are walking, walking upstairs, walking downstairs, sitting, standing and lying. It involved three stages of activity recognition; namely, data signal processing (filtering and segmentation), feature extraction and classification. Five types of ensemble classifiers utilized are Bagging, Adaboost, Rotation forest, Ensembles of nested dichotomies (END) and Random subspace. These ensemble classifiers employed Support vector machine (SVM) and Random forest (RF) as the base learners of the ensemble classifiers. The data classification is evaluated with the holdout and 10-fold cross-validation evaluation methods. The performance of each human daily activity was measured in terms of precision, recall, F-measure, and receiver operating characteristic (ROC) curve. In addition, the performance is also measured based on the comparison of overall accuracy rate of classification between different ensemble classifiers and base learners. It was observed that overall, SVM produced better accuracy rate with $99.22 \%$ compared to $\mathrm{RF}$ with $97.91 \%$ based on a random subspace ensemble classifier.
\end{abstract}

Keywords: gait; human activity recognition; smartphone; wearable sensor; human daily activity; ensemble method

\section{Introduction}

Recently, increasing interest has been shown in analysing human gait using wearable sensors, which is known as Human Activity Recognition (HAR). HAR with automatic recognition of physical activities is increasingly being studied and applied in Human-Computer Interaction (HCI), mobile and pervasive computing. One of the objectives of HAR is to offer information that enables computing frameworks to productively aid users in their tasks [1]. A few feasible applications that can be used with HAR which improves the service are on-request data systems, monitoring and surveillance system of smart homes, interactive interfaces for mobile services and games, and medical care service applications for both inpatient and outpatient treatments [2-4]. In addition, other applications include bilateral links targeted to advertising, entertainment, games, and multimedia visualization guidance $[5,6]$. 
Typically, human daily activities are divided into three categories, namely, gestures, low-level activities and high-level activities. Gestures involve simple activities such as the opening-closing of hands and bending of arms. The low-level activities are standing, sitting, walking, cycling and jogging, whereas, the high-level activities are cooking, dancing, eating, drinking and talking. A number of researchers have explored machine vision systems in gesture and activity recognition from video and still images in various settings [7-9].

Advances in sensor innovation in on-body wearable sensors and smartphones have enabled them to be used effectively for HAR systems. However, the difference between on-body wearable sensor-based HAR systems and the smartphone-based HAR systems is that, smartphones have a few integrated sensors that are equipped to provide an extensive variety of selections embedded into one cohesive device. Moreover, smartphones have computing ability, although not as capable as the devoted control units of wearable-sensor systems. In addition, smartphones have become an essential gadget in human's daily life and the usage of a smartphones greatly exceeds that of on-body wearable sensor-based systems. Hence, smartphones have become a prominent tool for assisting and supporting patients undergoing health rehabilitation and treatment, activity monitoring of daily living and diets, and for numerous other health issues [10,11].

One of the critical issues in HAR is the classification of the different activities performed by the users. The studies conducted in past show that machine learning algorithms such as Naïve Bayes tree (NBTREE), Decision Trees (C4.5), Neural Network (NN), k-Nearest Neighbour (k-NN) and Support Vector Machine (SVM) have been used for classification employing smartphone data [12-15]. Recently, Ensemble learning with bagging and boosting techniques have been found to enhance the accuracy of classifiers. Ensemble learning has been effectively tested and validated adequately on various datasets $[16,17]$.

The study described in this paper highlights the classification of six different daily activities based on the data acquired from inertial sensors of a smartphone using ensemble methods such as Bagging, Adaboost, Rotation forest, Ensemble nested dichotomies (END) and Random subspace together with two base learner techniques such as SVM and Random forest (RF). Two datasets from the UCI Machine Learning Repository [18] were utilised in the study, where each dataset involved 30 subjects performing activities such as walking, walking upstairs, walking downstairs, sitting, standing and laying. The classifications have been evaluated in terms of accuracy, precision, recall, F-measure, and receiver operating characteristic (ROC) curve. This paper is structured as follows: Section 2 discusses the literature review in terms of related work and general HAR system. Methods are presented in Section 3, followed by Section 4 which describes the results and discussion, and finally, conclusions are provided in Section 5.

\section{Literature Review}

\subsection{Related Work}

Over the last few years, from 2013 to 2018, human daily activities such as sitting on chairs and on the floor, lying right, lying left, slow walk, brisk walk, walking upstairs, walking downstairs, standing, laying, etc. have been extensively studied. Many researchers have worked towards evaluation of HAR using the smartphone with different daily activities. These activities are classified based on feature extraction schemes that are broadly categorized as time and frequency domains. In [19-25] researchers have implemented the time domain and frequency domain feature extraction as a combined approach. Other researchers in [26-31] have used feature extraction in the time domain only, whilst, in [32] researchers applied the frequency domain and time-frequency domain. The authors in [33] have chosen the time domain, frequency domain and time-frequency domain for feature extraction. Out of these studies, Saha et al. [19] found that the ensemble classifier performs best, with an overall accuracy rate of $94 \%$ using accelerometer and gyroscope sensor data. In the research carried out by Mohamed et al. [20], a combination of accelerometer data from the arm, belt and pocket analysed using 
rotation forest with the base learner $\mathrm{C} 4.5$, was found to provide the best overall classification accuracy rate of 98.9\% [20]. Researchers in [21,32], and [23-25] have analyzed the same dataset. Ronao and Cho [21] found that classification using two stages of continuous hidden Markov model (TS-CHMM) achieved the highest overall accuracy rate of 93.18\%. Jiang, Yin et al. [32] have obtained the best overall accuracy rate of $97.59 \%$ using deep convolution neural network (DCNN). Research reported by Kastner et al. [23] provided the best results with an overall classification accuracy rate of $96.23 \%$ with generalized learning vector quantization (GLVQ). Romero et al. [24] have found that One vs. one (OVO), OVO-SVM gives the best overall classification accuracy rate as 96.4\%; whilst, Anguita et al. [25] managed to gain a little improvement and reported best overall accuracy rate as $96.5 \%$ for classification using One vs. all (OVA), OVA-SVM. Researchers in [26-28], have analyzed the same dataset for walking, jogging, walking downstairs, walking, upstairs, sitting, and standing activities. A study conducted by Sufyan et al. [26] found that classification on voting Multilayer perceptron (MLP) and NBtree give the best accuracy rate for classification based on each activity. This study found that Voting MLP-NBtree gives the best accuracy rate of classification on walking at 99.23\%, jogging at $98.86 \%$, walking upstairs at $93.35 \%$, walking downstairs at $90.15 \%$, sitting at $98.37 \%$ and standing at 98.37\%. Research by Daghistani and Alshammari [27] found the best overall classification accuracy rate using Adaboost (J48) at 94.034\%, whilst, Catal et al. [28] reported the highest overall classification accuracy rate based on voting (J48, logistic regression and MLP) as 94.06\%. Gupta and Kumar [29] stated the study on human activities on sitting, standing, walking and running produced the best overall accuracy rate of $98.83 \%$ using an Adaboost classifier. Research by Gao et al. [33] showed that C4.5 was the best classifier with a $96.4 \%$ overall accuracy rate for lying, sitting, standing, walking and transition activity. Bayat et al. [30] studied HAR comparison between different classifiers such as MLP, SVM, RF, simple logistic, logitboost, Logistic model tree (LMT) and voting classifier with the triaxial accelerometer data in a smartphone that placed in pocket and hand. The results of this study showed that data in hand produces best overall classification accuracy rate using voting combination of MLP, logitboost and SVM classifier with $91.15 \%$, whilst, data in pocket gives best accuracy of classification rate as $90.34 \%$ using voting combination of MLP, RF and simple logistic. Research studies from Ha and Ryu [31] have reported that the best overall classification accuracy rate of $97.8 \%$ was obtained with an ensemble method known as Error correcting output coding (ECOC) that was combined with the random forest as the base learner. Overall, it can be concluded that Ensemble methods produce better results compared with other algorithms.

\subsection{HAR System}

HAR is a way toward recognizing common human activities in daily living. It is turning into an attractive research field because of numerous areas of application. Physiological signals, environmental signals, location data and acceleration signals are the categories of input data that are acquired from wearable sensors in an HAR setting. Physiological signals data such as skin conductivity, heart rate, skin temperature, respiration rate and electrocardiography signals have also been considered in a few research studies to improve the recognition accuracy [34]. Environmental signals, for example, audio level, temperature, humidity are proposed to provide information exposing the individual's environment [35,36]. The Global Positioning System (GPS) empowers all kinds of area-based information. Current smartphones are equipped with integrated GPS, making it exceptionally advantageous for context-aware applications such as the recognition of the individual's transportation mode [37]. Accelerometers can be categorized into two types: either as body wearable sensors or incorporated with new models of mobile devices such as smartphones and smart watches which record the body movement [38,39]. The majority of research work in HAR applications is performed using wearable sensors. Figure 1 shows the HAR with different wearable sensors. 


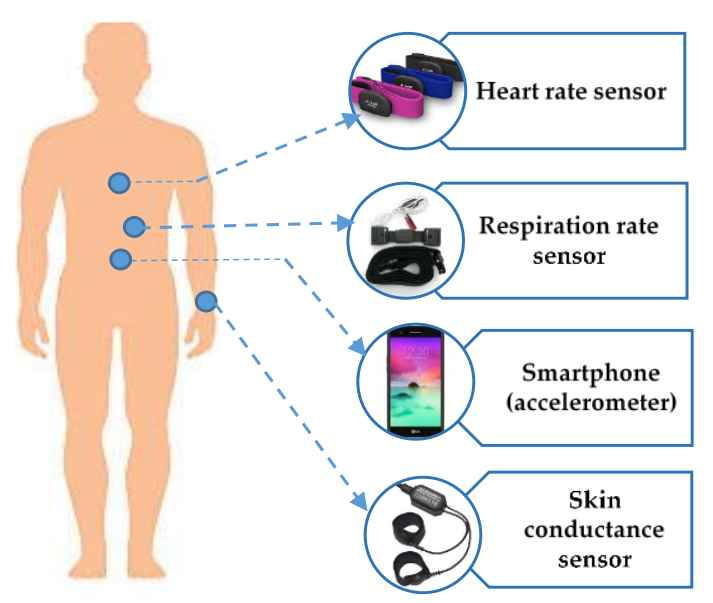

Figure 1. Wearable sensors for human daily activities.

The various types of wearable sensors are used to identify different human activities in three categories: gesture, low-level activities and high-level activities as shown in Figure 2.

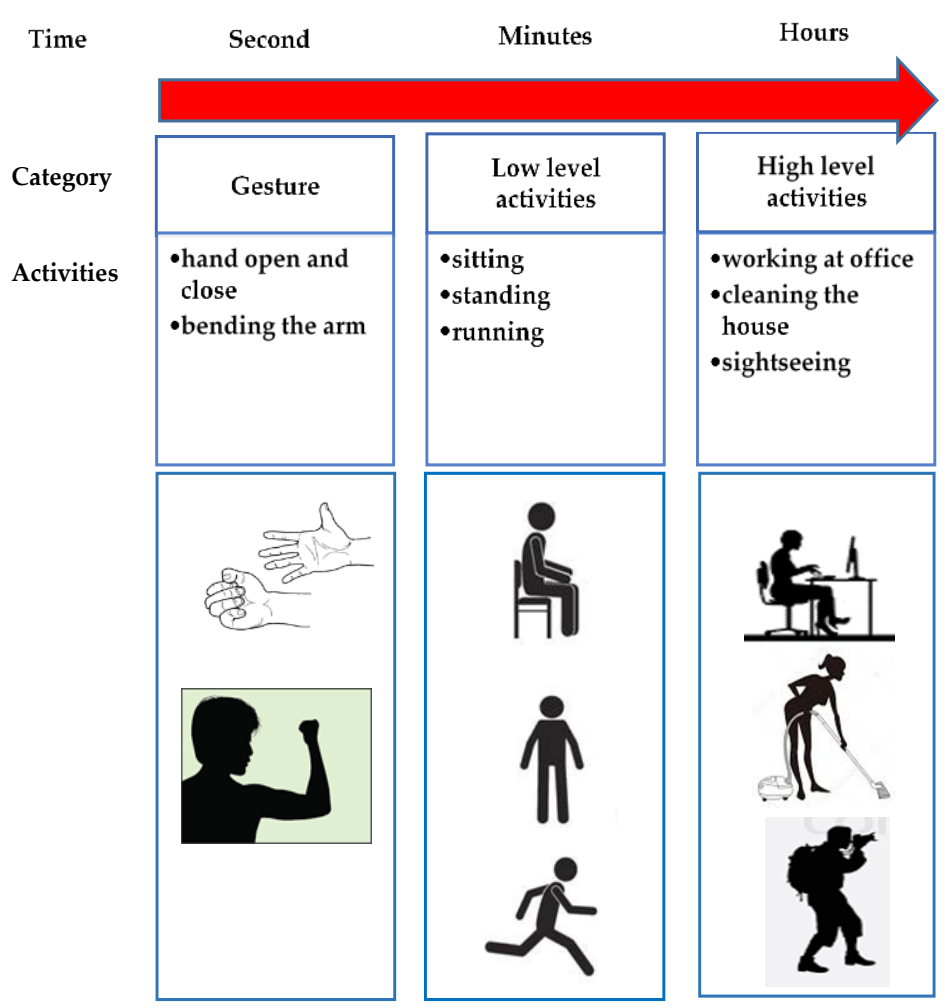

Figure 2. Categories of human daily activities.

Gestures activities are classified as extremely short activities such as bending the arm, or opening and closing the hands. Human daily activities such as sitting, standing and running which typically last between seconds and a few minutes are known as low-level activities. Activities like working at the office, cleaning the house and sightseeing normally last for more than a few minutes up to a few hours are known as high-level activities [40]. Detailed information about the general process for training and testing the data of HAR systems based on wearable sensors can be found in [41]. 


\section{Methods}

\subsection{Signal Processing Method for HAR}

The signal processing method of HAR system that was carried out in this research is shown in Figure 3.

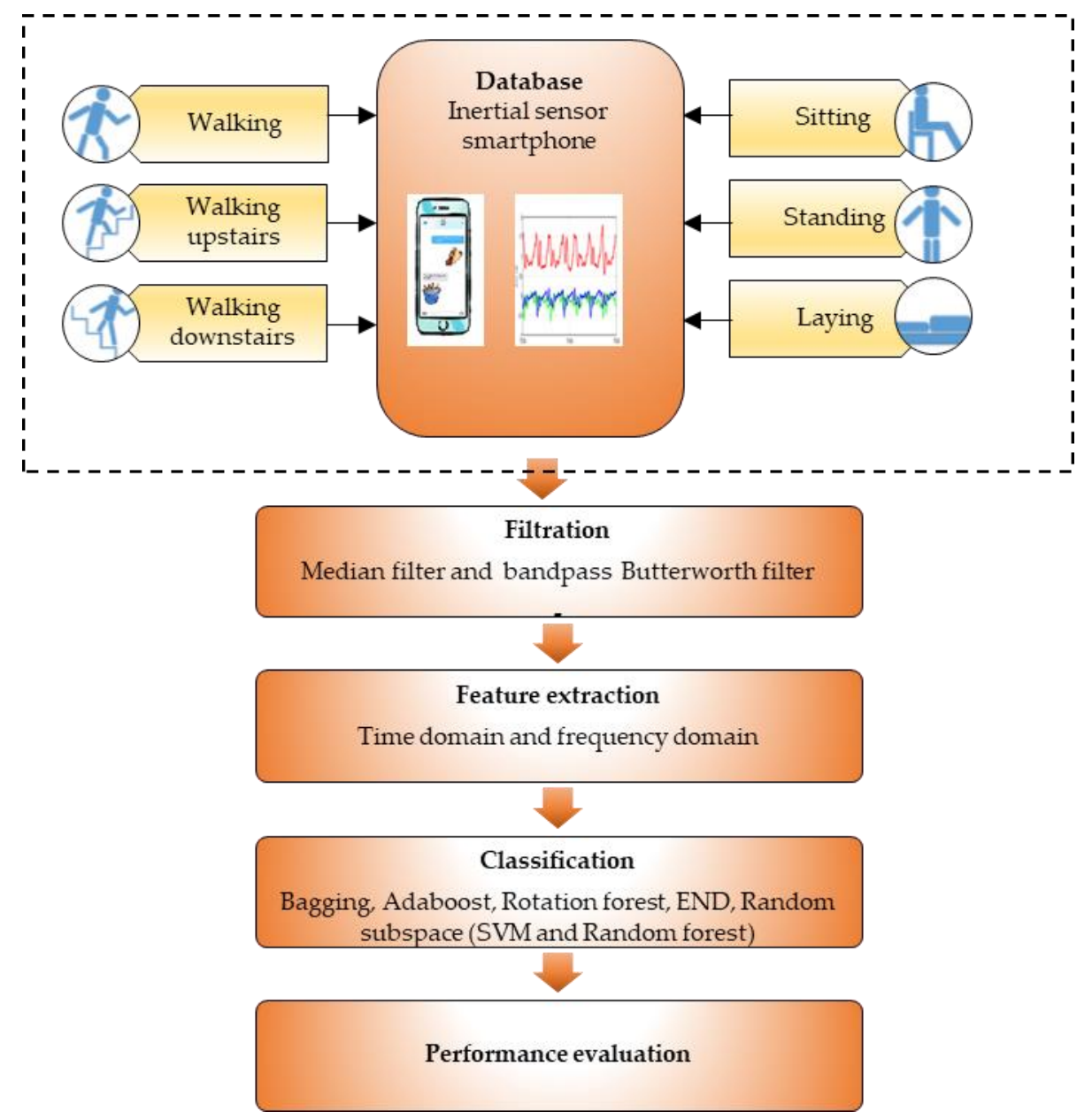

Figure 3. Signal processing method of HAR system.

According to Figure 3, the raw signals of human daily activities such as walking, walking upstairs, walking downstairs, sitting, standing and laying are acquired from the inertial sensors of smartphones. Then, the raw signals are segmented using sliding windows and filtered using median and band pass Butterworth filters to remove the irrelevant information or noise. Next, feature extraction of time and frequency domain are implemented. Thereafter, all the data of extracted features are classified with ensemble classifiers using base learners of SVM and RF. The performance of classification of is measured using performance evaluation metrics such as precision, recall, F-measure, accuracy and ROC.

\subsection{Database}

For this study, two datasets were obtained from UCI Machine Learning Repository [18]. The datasets consist of dataset 1 and dataset 2, containing 5447 data samples and 10,299 data samples, respectively. Dataset 1 was collected from a smartphone worn around the waist of 31 participants within the age group of 22-79 years. Each activity was performed for $60 \mathrm{~s}$. For dataset 2, a group of 30 volunteers with ages ranging 19-48 years were selected. Each person was instructed to follow 
a protocol of activities while wearing a waist mounted smartphone. For both datasets, the data collections were performed using Samsung Galaxy S2 smartphones which comprise an accelerometer and gyroscope to acquire tri-axial linear acceleration and angular velocity signals at a sampling rate of $50 \mathrm{~Hz}$. Although the tasks were carried out in laboratory conditions, volunteers were asked to perform freely the sequence of activities aiming to simulate a more naturalistic dataset. Table 1 shows the activities that were carried out for both the datasets.

Table 1. List of daily living activities.

\begin{tabular}{cc}
\hline Activity Reference & Description of Activity \\
\hline A1 & Walking \\
A2 & Walking upstairs \\
A3 & Walking downstairs \\
A4 & Sitting \\
A5 & Standing \\
A6 & Laying \\
\hline
\end{tabular}

\subsection{Data Pre-Processing Filtering and Feature Extraction}

The raw signals were processed by applying a median filter and 3rd order low pass Butterworth filter with $20 \mathrm{~Hz}$ cut-off. These signals were then segmented with fixed width sliding windows of $2.56 \mathrm{~s}$ with $50 \%$ overlap. A total of 17 features extracted from the time and frequency domains for each window were used in this work, where only about six features are shown in Table 2 . The full 17 features can be found in [42].

Table 2. 6 Features extraction of time and frequency domain for each window.

\begin{tabular}{cc}
\hline Feature & Description \\
\hline Min & Smallest value in the array \\
Max & Largest value in the array \\
Std & Standard deviation \\
Entropy & Signal entropy \\
Kurtosis & Kurtosis of the frequency domain signal \\
Skewness & Skewness of the frequency domain signal \\
\hline
\end{tabular}

\subsection{Classification Techniques-Ensemble Methods}

The principle thought behind the ensemble method is to evaluate a few single classifiers and combine them to acquire a classifier that surpasses each individual one of them. The motivation behind supervised learning is to classify patterns or instances that are specified into a group denoted as labels or classes. Often, the classification is determined by a classification models (classifiers) that are inferred from a pre-classified design model. Nevertheless, the classification employs knowledge that is provided by a specialist in the application area which is referred to as training data. The training set is a standardized supervised learning set that has a set of instances. The labels of the instances in the training set are identified and the objective is to develop a model with a specific end goal to label new instances. An algorithm which builds the model is called inducer and a case of an inducer for a specific training set is known as a classifier [43]. An ensemble comprises several inducers which are commonly referred to as the base classifier or base learner. A base learner is an algorithm that receives a set of labelled instances as input and produces a model that simplifies these instances. Predictions are determined for new unclassified instances by utilizing the created model. The generalization capability of an ensemble is usually more robust than that of base learners. As a matter of fact, ensemble method is likable because it can boost weak learners, referred to base classifiers which are marginally better than random estimate to strong learners which can make very accurate predictions. In this research work, ensemble methods such as Bagging, Adaboost M1, Rotation forest, Ensembles of 
Nested Dichotomies (END) and random subspace were used for classification. An ensemble inducer can be of any type of base classifiers such as decision tree, neural network, k-NN and others type of base learner algorithm [43]. In this research work, the base learners applied were SVM and RF. The detail information about the ensemble methods can be found in [44] for bagging, [45,46] for Adaboost.M1, [47] for Rotation Forest, [48] for Ensembles Nested Dichotomies (END), [49] for Random subspace, [50] for Random Forest (RF) and [51] for SVM.

The above stated five Ensemble methods and two base learner algorithms were used to classify six human daily activities based on a classifier tool known as WEKA 3.8 Version [52] with model evaluation of the holdout method (contains $70 \%$ of the training set and $30 \%$ of the test set) and 10 -fold cross-validation method. A Wilcoxon test was performed on the results to discover if the accuracy rate of classification instances was significantly different for SVM as a baseline compared to the RF as base learners in five different ensemble methods. This statistical test was conducted on IBM SPSS version 20 [53]. A value of $p$ less than 0.05 is considered as statistically significant when the confidence level is set to $95 \%$.

\subsection{Performance Evaluation}

The performance evaluation that was implemented in this study is based on the following expressions:

$$
\begin{gathered}
\text { Precison }=\frac{\mathrm{TP}}{\mathrm{TP}+\mathrm{FP}} \\
\text { Recall }=\frac{\mathrm{TP}}{\mathrm{TP}+\mathrm{FN}} \\
\mathrm{F}-\text { measure }=\frac{2(\mathrm{TP})}{2((\mathrm{TP})+(\mathrm{FN}))} \\
\text { Accuracy }=\frac{\mathrm{TP}+\mathrm{TN}}{\mathrm{TP}+\mathrm{TN}+\mathrm{FP}+\mathrm{FN}}
\end{gathered}
$$

Receiver operating characteristic (ROC) curve is a plot graph of the true positive rate against the false positive rate at different classification threshold setting. The true positive rate is also known as Recall in Equation (2) and false positive is defined as following expression:

$$
\text { False positive rate }=\frac{\mathrm{FP}}{\mathrm{FP}+\mathrm{TN}}
$$

where TP—True Positive, TN—True Negative, FP—False Positive, and FN—False Negative.

\section{Results and Discussion}

\subsection{Performance Evaluation of Dataset 1}

\subsubsection{Holdout and 10 Cross-Validations for Precision, Recall, F-measure, and ROC Evaluation}

Table 3 presents the performance evaluation of END classifier with SVM and RF as base learner including precision, recall, F-measure and receiver operating characteristic (ROC) as the best classifier of the holdout method.

As shown in Table 3, the results of END classifier evaluation on holdout method obtained the best precision in activity A6 with 100\% using SVM as a base learner compared to RF at $99.1 \%$. For activity A5, both SVM and RF base learners achieved the precision results at $88.8 \%$. But in activity A4, better precision was obtained by RF with $96.6 \%$ followed by SVM with $92.8 \%$. For A3, A2 and A1, precision results show that SVM has given higher result range of $95.3 \%$ to $96.1 \%$ compared to RF with $89.6 \%$ to $92.7 \%$. Recall results show that SVM obtained $99.10 \%$ and RF obtained $97.30 \%$ for activity A6. However, for activity A5, RF produced $94.6 \%$ compared to SVM with 91.5\%. For A4, A3, A2, and A1, recall results show that SVM produced results ranging from $90.4 \%$ to $97.6 \%$ compared to RF with $90.1 \%$ 
to $94.9 \%$. Results of F-measure evaluation for activity A6 is 99.6\% for SVM and 98.2\% for RF. However, the F-measure results for activity A4 and A5 for RF are 93.2\% and 91.6\% respectively compared to SVM with $91.60 \%$ and $90.20 \%$. Activities A1, A2, and A3 give SVM better F-measure results ranging from $94.7 \%$ to $96.9 \%$, higher than RF range from $90.2 \%$ to $93.8 \%$. RF gained greater results for ROC evaluation with results ranging from $99.4 \%$ to $100 \%$ compared to SVM which produced $95.2 \%$ to $99.8 \%$ for all activities. Table 4 presents the performance evaluation of Random subspace classifier with SVM and RF as base learner for 10-fold cross-validation method.

Table 3. Performance evaluation of each activity with random subspace classifier on the holdout method.

\begin{tabular}{|c|c|c|c|c|c|c|c|c|}
\hline \multicolumn{9}{|c|}{ END (Holdout) } \\
\hline \multicolumn{5}{|c|}{ SVM } & \multicolumn{4}{|c|}{ RF } \\
\hline Activity & Precision & Recall & F-measure & ROC & Precision & Recall & F-measure & ROC \\
\hline A1 & $96.10 \%$ & $97.60 \%$ & $96.90 \%$ & $99.20 \%$ & $92.70 \%$ & $94.90 \%$ & $93.80 \%$ & $99.70 \%$ \\
\hline A2 & $95.30 \%$ & $94.00 \%$ & $94.70 \%$ & $98.80 \%$ & $89.60 \%$ & $90.80 \%$ & $90.20 \%$ & $99.40 \%$ \\
\hline A3 & $95.50 \%$ & $96.00 \%$ & $95.70 \%$ & $98.80 \%$ & $92.30 \%$ & $91.90 \%$ & $92.10 \%$ & $99.50 \%$ \\
\hline A4 & $92.80 \%$ & $90.40 \%$ & $91.60 \%$ & $95.20 \%$ & $96.60 \%$ & $90.10 \%$ & $93.20 \%$ & $99.60 \%$ \\
\hline A5 & $88.80 \%$ & $91.50 \%$ & $90.20 \%$ & $96.20 \%$ & $88.80 \%$ & $94.60 \%$ & $91.60 \%$ & $99.40 \%$ \\
\hline A6 & $100.00 \%$ & $99.10 \%$ & $99.60 \%$ & $99.80 \%$ & $99.10 \%$ & $97.30 \%$ & $98.20 \%$ & $100.00 \%$ \\
\hline
\end{tabular}

Table 4. Performance evaluation for each activity of a random subspace classifier on 10 -fold cross-validation method.

\begin{tabular}{|c|c|c|c|c|c|c|c|c|}
\hline \multicolumn{9}{|c|}{ Random Subspace (10-fold Cross-Validation) } \\
\hline \multicolumn{5}{|c|}{ SVM } & \multicolumn{4}{|c|}{ Random Forest } \\
\hline Activity & Precision & Recall & F-measure & ROC & Precision & Recall & F-measure & ROC \\
\hline A1 & $95.60 \%$ & $97.70 \%$ & $96.70 \%$ & $99.30 \%$ & $90.30 \%$ & $95.90 \%$ & $93.10 \%$ & $99.60 \%$ \\
\hline $\mathrm{A} 2$ & $95.40 \%$ & $94.80 \%$ & $95.10 \%$ & $98.70 \%$ & $92.50 \%$ & $90.00 \%$ & $91.20 \%$ & $99.30 \%$ \\
\hline A3 & $96.60 \%$ & $94.70 \%$ & $95.70 \%$ & $98.30 \%$ & $93.80 \%$ & $90.10 \%$ & $91.90 \%$ & $99.40 \%$ \\
\hline A4 & $93.00 \%$ & $93.90 \%$ & $93.40 \%$ & $98.20 \%$ & $96.40 \%$ & $94.20 \%$ & $95.30 \%$ & $99.80 \%$ \\
\hline A5 & $93.50 \%$ & $92.50 \%$ & $93.00 \%$ & $98.60 \%$ & $94.10 \%$ & $95.90 \%$ & $95.00 \%$ & $99.60 \%$ \\
\hline A6 & $99.00 \%$ & $99.40 \%$ & $99.20 \%$ & $99.80 \%$ & $98.00 \%$ & $98.20 \%$ & $98.10 \%$ & $100.00 \%$ \\
\hline
\end{tabular}

As shown in Table 4, the results of Random subspace classifier using 10-fold cross-validation indicates SVM as base learner produces better precision range of results from $95.6 \%$ to $99 \%$ compared to RF with $90.3 \%$ to $98 \%$ for activities A1, A2, A3 and A6. However, RF has obtained better precision results of $94.2 \%$ and $95.9 \%$ compared to SVM with $93 \%$ and $93.5 \%$ for activities A4 and A5. For recall, activities A1, A2, A3, and A6 with SVM produced superior results from $94.7 \%$ to $99.4 \%$ compared to RF with $90 \%$ to $98.2 \%$. However, RF has obtained better recall results of $94.2 \%$ and $95.9 \%$ compared to SVM with $93.9 \%$ and $92.5 \%$ for activities A4 and A5. The results of F-measure for activities A1, A2, A3 and A6 ranged from 95.1\% to 99.2\% for SVM and 91.2\% to 98.1\% for RF. The ROC results for RF were from $99.3 \%$ to $100 \%$ and $98.2 \%$ to $99.8 \%$ for SVM for all the activities. In dataset 1 , overall results of 10-fold cross-validation model evaluation give better results compared to holdout for both base learners.

\subsubsection{Holdout and 10-Fold Cross-Validations for Overall Accuracy Rate}

Figures 4 and 5 show the accuracy rate classification for each activity for ensemble method with base learners SVM and RF using holdout and 10-fold cross validation methods, respectively, for dataset 1 .

As shown in Figure 4, the accuracy rate of activity A1, A2, A3 and A6 of Random subspace classifier with SVM for holdout method produced superior accuracy rate with the range from $98.4 \%$ to $99.9 \%$ compared to RF which produced an accuracy rate of $97.0 \%$ to $99.4 \%$. On the other hand, RF produced better results with an accuracy rate of $97.1 \%$ and $96.9 \%$ compared to SVM with $97.1 \%$ and $96.9 \%$ activities $\mathrm{A} 4$ and $\mathrm{A} 5$. 


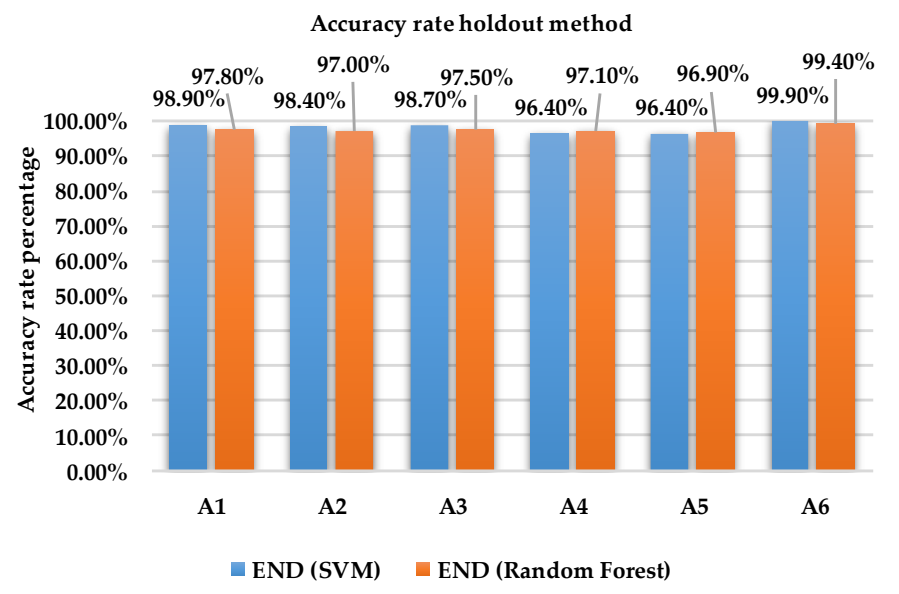

Figure 4. Accuracy rate of each activity holdout method dataset 1.

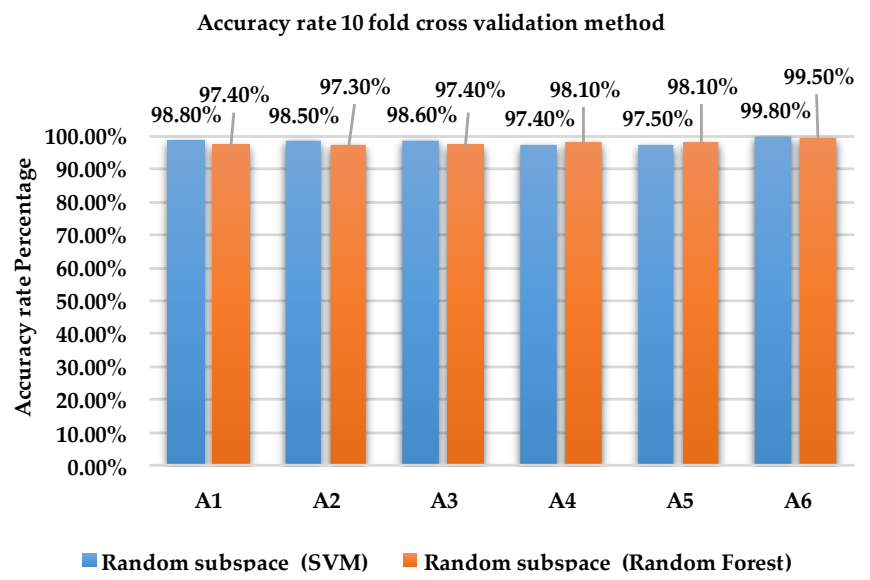

Figure 5. Accuracy rate of each activity 10 fold cross validation method dataset 1 .

Results from Figure 5 indicate that the accuracy rate for activities A1, A2, A3 and A6 of Random subspace classifier with SVM using 10-fold cross validation is higher with $98.5 \%$ to $99.8 \%$ compared to RF with $97.3 \%$ to $99.5 \%$. The accuracy rate for activities A4 and A5 is $97.4 \% 97.5 \%$ for SVM and $98.1 \%$ for RF.

Tables 5 and 6 show the overall accuracy rate of all other ensemble methods with base learners SVM and RF using holdout method and 10-fold cross validation method for dataset 1 .

Table 5. Overall performance evaluation ensemble methods on the holdout method.

\begin{tabular}{cccc}
\hline \multicolumn{4}{c}{ Overall Accuracy Rate } \\
\hline Ensemble Method & Holdout & & \\
\hline SVM & RF & $p$-Value \\
Adabging & $93.83 \%$ & $91.62 \%$ & 0.028 \\
Rotation forest & $94.24 \%$ & $94.24 \%$ & 0.917 \\
END & $89.95 \%$ & $92.23 \%$ & 0.344 \\
Random subspace & $94.50 \%$ & $93.16 \%$ & 0.172 \\
\hline
\end{tabular}

Results in Table 5 demonstrate that SVM has a significantly greater accuracy with Bagging $(93.83 \%$, $p=0.028$ ). There were no significant differences for other ensemble methods using the holdout method and 10-fold cross-validation method as shown in Table 6 for dataset 1. 
Table 6. Overall performance evaluation ensemble classifiers for 10-fold cross-validation method.

\begin{tabular}{cccc}
\hline \multicolumn{3}{c}{ Overall Accuracy Rate } \\
\hline Ensemble Method & 10-Fold Cross-Validation & \\
& SVM & Random Forest & $p$-Value \\
\hline Bagging & $94.57 \%$ & $92.88 \%$ & 0.173 \\
Adaboost & $94.84 \%$ & $94.74 \%$ & 0.917 \\
Rotation forest & $90.65 \%$ & $93.65 \%$ & 0.075 \\
END & $95.14 \%$ & $94.48 \%$ & 0.249 \\
Random subspace & $95.33 \%$ & $94.08 \%$ & 0.249 \\
\hline
\end{tabular}

\subsection{Performance Evaluation of Dataset 2}

\subsubsection{Holdout Method for Precision, Recall, F-measure, and ROC Evaluation}

Table 7 presents the performance evaluation of random subspace classifier with SVM and RF as base leaners as the best classifier of holdout method.

Table 7. Performance evaluation of each activity with random subspace classifier on the holdout method.

\begin{tabular}{ccccccc}
\hline \multicolumn{7}{c}{ Random Subspace (Holdout) } \\
\hline Activity & Precision & Recall & F-measure & Precision & $\begin{array}{c}\text { RF } \\
\text { Recall }\end{array}$ & F-measure \\
\hline A1 & $99.80 \%$ & $100.00 \%$ & $99.90 \%$ & $98.20 \%$ & $99.00 \%$ & $98.60 \%$ \\
A2 & $98.90 \%$ & $99.50 \%$ & $99.20 \%$ & $98.20 \%$ & $98.60 \%$ & $98.40 \%$ \\
A3 & $99.80 \%$ & $99.00 \%$ & $99.40 \%$ & $98.80 \%$ & $97.30 \%$ & $98.00 \%$ \\
A4 & $96.70 \%$ & $97.20 \%$ & $97.00 \%$ & $96.60 \%$ & $95.30 \%$ & $95.90 \%$ \\
A5 & $97.70 \%$ & $97.00 \%$ & $97.30 \%$ & $95.80 \%$ & $97.20 \%$ & $96.50 \%$ \\
A6 & $100.00 \%$ & $100.00 \%$ & $100.00 \%$ & $100.00 \%$ & $99.80 \%$ & $99.90 \%$ \\
\hline
\end{tabular}

As shown in Table 7, the results of random subspace classifier evaluation on holdout method obtained the best precision in activity A6 with 100\% for SVM and RF. For other activities, SVM shows better precision results between $96.7 \%$ and $99.8 \%$ compared to RF which obtained $95.8 \%$ to $98.8 \%$. For recall, SVM achieved 100\% compared to RF 99.8\% for activity A6. SVM results for activities A1, A2, A3 and A4 ranges from 97\% to 100\% compared RF which obtained $95.8 \%$ to $99 \%$. However, RF produced better recall results with $97.2 \%$ compared to SVM which recorded 97\% for activity A5. The F-measure evaluation shows that SVM obtained 100\% compared to RF that produced $99.9 \%$ for activity A6. For activity A4, SVM and RF obtained 97\% and 95.9\% respectively which are the lowest performance compared to activities A1, A2, A3 and A5. Table 8 shows the ROC curve of each activity of Random subspace with SVM classifier and Random subspace with RF using hold out method for dataset 2.

As shown in Table 8, the results of ROC evaluation gained 1.000 in activities A1, A2, A3 and A6 in both base learners. For activities A4 and A5, the ROC results for RF was 0.999 compared to 0.995 and 0.998 for SVM.

Table 8. ROC for each activity of the random subspace classifier on holdout method.

\begin{tabular}{ccc}
\hline \multicolumn{3}{c}{ Random Subspace (Holdout) } \\
\hline & SVM & RF \\
Activity & ROC & ROC \\
\hline A1 & 1.000 & 1.000 \\
A2 & 1.000 & 1.000 \\
A3 & 1.000 & 1.000 \\
A4 & 0.995 & 0.999 \\
A5 & 0.998 & 0.999 \\
A6 & 1.000 & 1.000 \\
\hline
\end{tabular}


4.2.2. 10-Fold Cross Validation for Precision, Recall, F-measure, and ROC Evaluation

The cross-validation method evaluates fold $(k)=10$ for all activities of dataset 2 . Table 9 presents the performance evaluation of Random subspace as the best classifier of 10-fold cross-validation method.

Table 9. Performance evaluation for each activity of the random subspace classifier on 10-fold cross-validation method. Random subspace (10-fold cross-validation method).

\begin{tabular}{ccccccc}
\hline \multicolumn{7}{c}{ Random Subspace (10-Fold Cross-Validation Method) } \\
\hline \multicolumn{7}{c}{ SVM } \\
Activity & Precision & Recall & F-measure & Precision & Recall & F-measure \\
\hline A1 & $99.90 \%$ & $100.00 \%$ & $100.00 \%$ & $99.90 \%$ & $98.40 \%$ & $98.70 \%$ \\
A2 & $99.70 \%$ & $99.70 \%$ & $99.70 \%$ & $97.50 \%$ & $99.20 \%$ & $98.30 \%$ \\
A3 & $99.70 \%$ & $99.80 \%$ & $99.80 \%$ & $98.50 \%$ & $97.60 \%$ & $98.00 \%$ \\
A4 & $97.90 \%$ & $98.00 \%$ & $98.00 \%$ & $97.00 \%$ & $95.20 \%$ & $96.10 \%$ \\
A5 & $98.20 \%$ & $98.10 \%$ & $98.10 \%$ & $95.60 \%$ & $97.30 \%$ & $96.40 \%$ \\
A6 & $100.00 \%$ & $100.00 \%$ & $100.00 \%$ & $100.00 \%$ & $99.80 \%$ & $99.90 \%$ \\
\hline
\end{tabular}

From Table 9, the precision results for random subspace classifier on 10-fold cross-validation method shows that SVM and RF obtained 99.9\% for activity A1 and 100\% for both the SVM and RF for activity A6. For activities A2, A3, A4, and A5, SVM obtained between 97.9\% and 99.9\% and RF 95.6\% to $98.5 \%$. Recall results achieved 100\% for activities A1 and A6 using SVM compared to RF which received $98.4 \%$ and $99.8 \%$. For activities A2, A3, A4, A5, SVM gained between 98\% and 99.8\%, whereas, RF obtained between $95.2 \%$ and $99.2 \%$. The results of F-measure for activities A1 and A6 for SVM was 100\% compared to RF which obtained between 98.7\% and 99.9\%. SVM obtained between $98 \%$ and $99.7 \%$ compared to RF which produced results between $96.1 \%$ and $98.3 \%$ for the results of F-measure in activities A2, A3, A4, and A5.

Table 10 shows the ROC of each activity of Random subspace with SVM and Random subspace with RF classifier using 10-fold cross validation method dataset 2.

Table 10. ROC for each activity of the random subspace classifier on 10-fold cross validation method.

\begin{tabular}{ccc}
\hline \multicolumn{2}{c}{ Random Subspace (10-Fold Cross Validation) } \\
\hline Activity & SVM & RF \\
A1 & ROC & ROC \\
A2 & 1.000 & 0.999 \\
A3 & 1.000 & 0.999 \\
A4 & 0.000 & 0.999 \\
A5 & 0.999 & 0.998 \\
A6 & 1.000 & 0.999 \\
\hline
\end{tabular}

As shown in Table 10, the results of ROC evaluation for activities A1, A2, A3, and A6 for SVM is 1.000, whereas, RF produced 1.000 only for A6. For activities A4 and A5, SVM recorded 0.999. RF achieved 0.998 for activity A4 and 0.999 for A1, A2, A3 and A5.

As can be seen in Tables 7-10, overall performance evaluation in each activity on Random subspace classifier with base learner SVM is better than RF for holdout method and 10-fold cross-validation methods. The 10-fold cross-validation model evaluation gives better results compared to holdout for both base learners.

Representative ROC curves for SVM and RF are shown in Figures 6 and 7 based on Table 10 for activities A1, A2 and A6. 


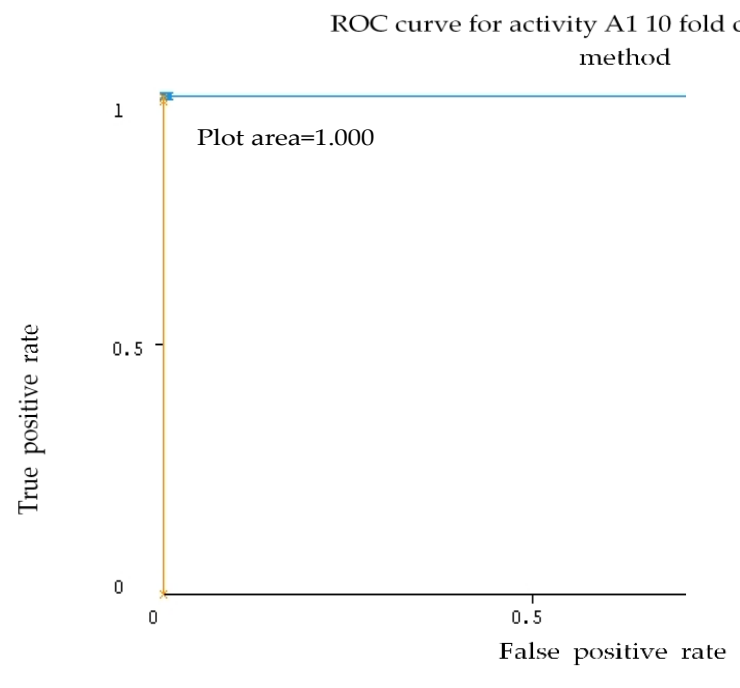

(a)

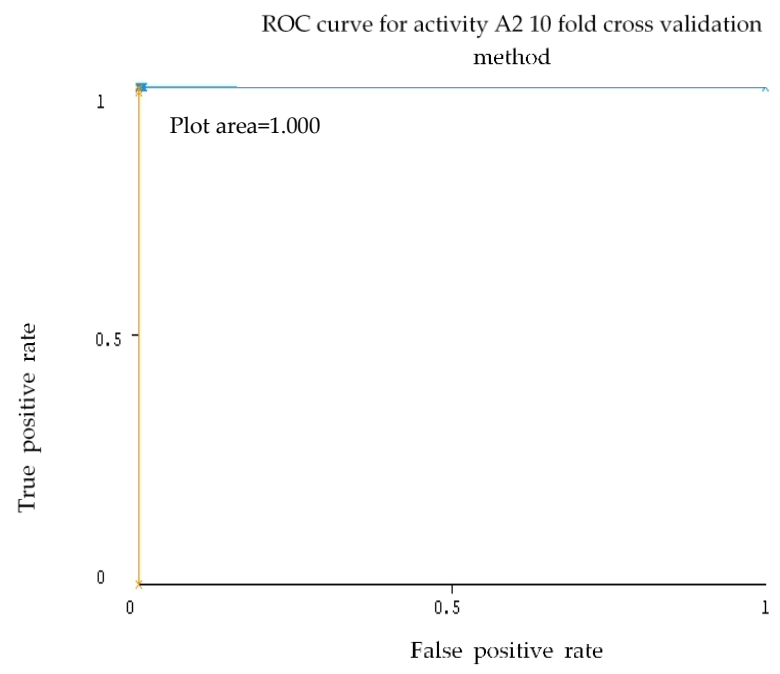

(b)

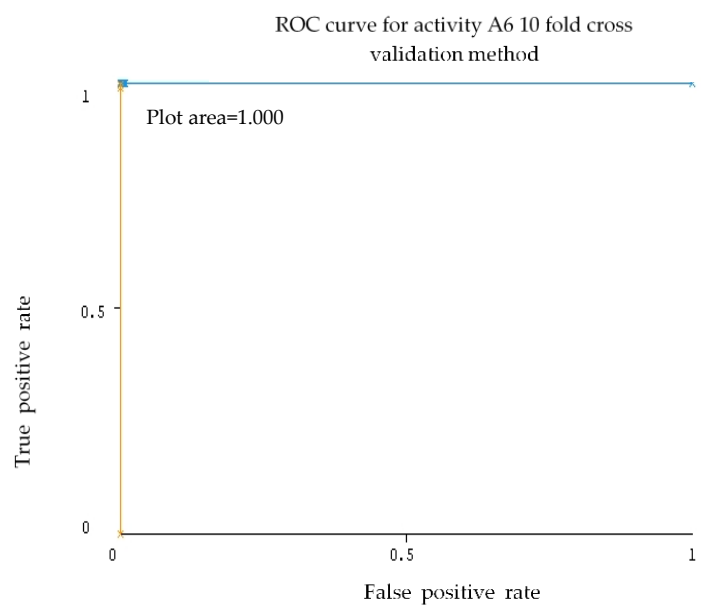

(c)

Figure 6. ROC graph activity A1 (a), A2 (b) and A6 (c) of Random subspace with SVM classifier using 10 fold cross validation method dataset 2 . 


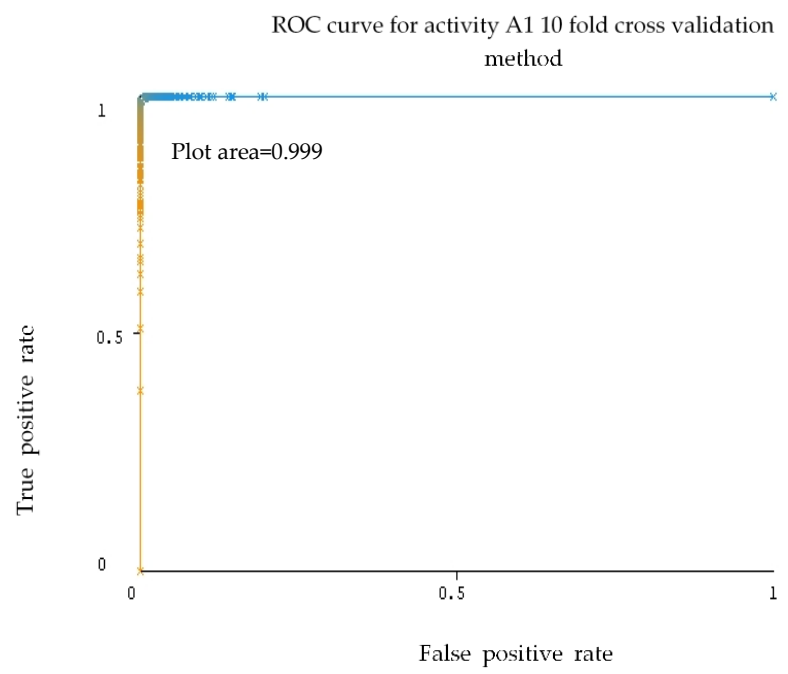

(a)

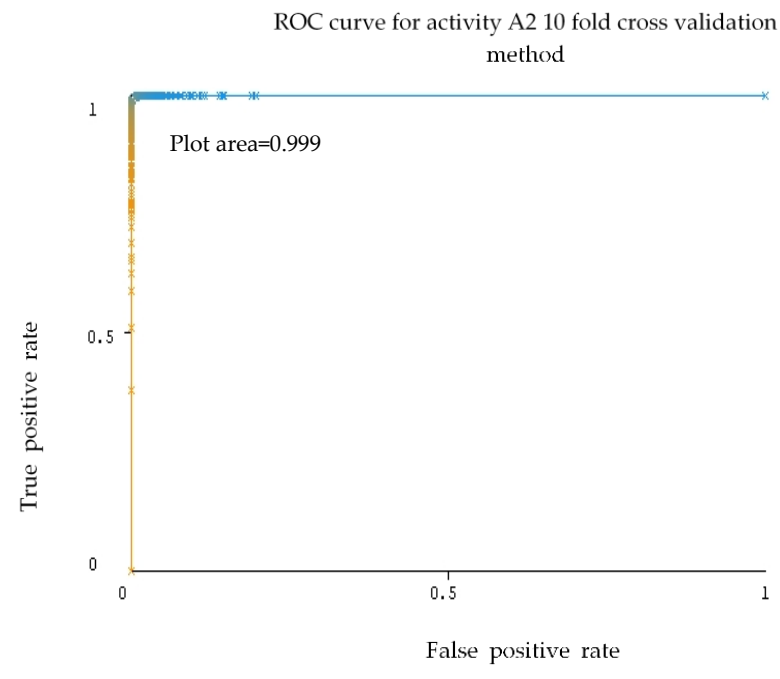

(b)

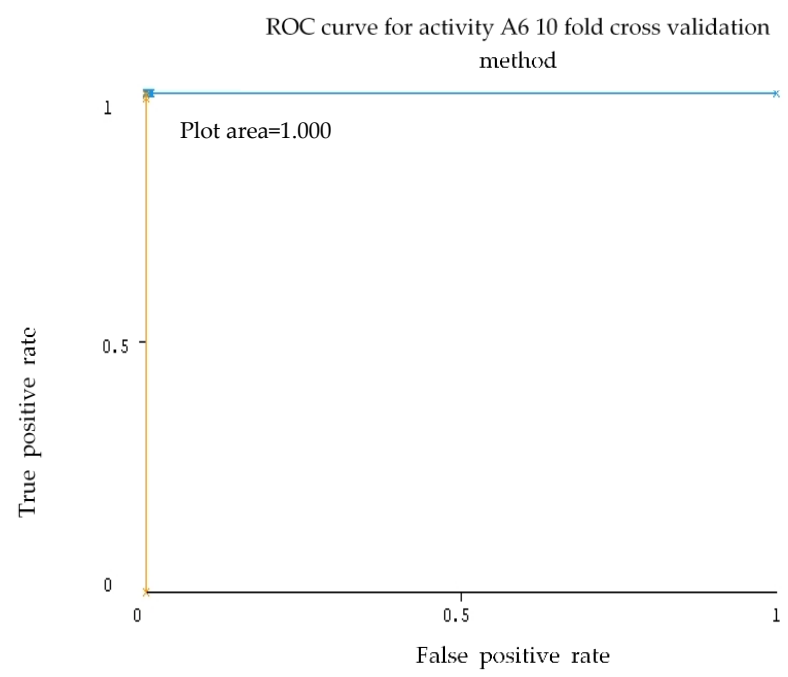

(c)

Figure 7. ROC graph activity A1 (a), A2 (b) and A6 (c) of Random subspace with RF classifier using 10 -fold cross validation method dataset 2 . 
As can be seen in Figures 6 and 7, the activities A1, A2 and A6 for SVM and RF, the ROC results produced 1.000. For activities A1 and A2, the ROC results are 0.999 for RF.

\subsubsection{Holdout and 10-Fold CROSS-validations for Overall Accuracy Rate Classification}

Figures 8 and 9 show the accuracy rate of classification for each activity for ensemble method with base learners SVM and RF using holdout method and 10-fold cross validation method for dataset 2.

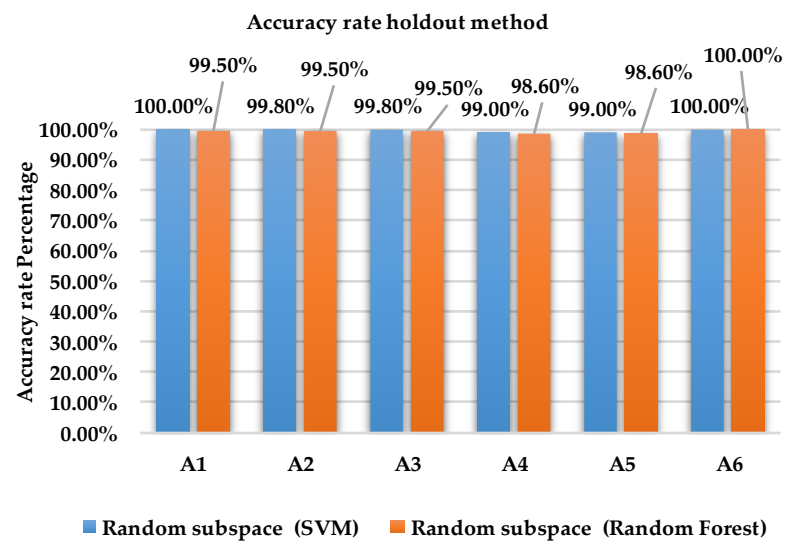

Figure 8. Accuracy rate of each activity for holdout method using dataset 2.

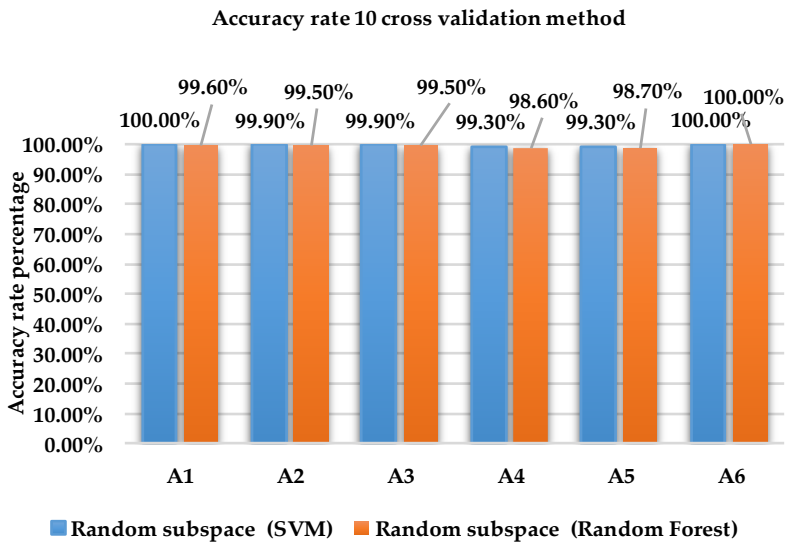

Figure 9. Accuracy rate of each activity of 10 -fold cross validation method using dataset 2.

As shown in Figure 8, the accuracy rate of activity A6 is 100\% for Random subspace classifier with SVM and RF using the holdout method. For activities A1, A2, A3, A4 and A5, the accuracy rate for SVM is between $99 \%$ and $100 \%$ compared to RF which obtained results between $98.6 \%$ and $99.5 \%$.

As shown in Figure 9, the accuracy rate of activity A6 is 100\% for Random subspace classifier with SVM and RF using the 10-fold cross validation method. For activities A1, A2, A3, A4 and A5, the accuracy rate for SVM is between $99.9 \%$ and $100 \%$ compared to RF which obtained results between $98.7 \%$ and $99.6 \%$.

Tables 11 and 12 present the overall accuracy rate classification of other ensemble methods with base learners SVM and RF on holdout method and 10-fold cross validation method for dataset 2.

Results in Table 11 show that SVM base learner has significantly greater accuracy rate than RF with the same probability value ( $p$-value) when using Bagging (98.54\%, $p=0.028)$, END (98.61\%, $p=0.028)$ and Random subspace $(98.74 \%, p=0.028)$. In this case, random subspace gives the highest accuracy rate in the holdout method. There was no significant difference in accuracy between the SVM and RF when employing Adaboost and RF for holdout method because of the higher $p$-value. From Table 12, SVM base learner demonstrates significantly greater accuracy rate than RF, whilst the 
random subspace gives the highest accuracy rate with $(99.22 \%, p=0.028)$. The results of END (99.20\%, $p=0.028)$, Adaboost (99.17\%, $p=0.028)$ and Bagging $(99.07 \%, p=0.028)$ have significantly greater overall accuracy rate than RF as a base learner in 10-fold cross-validation method.

Table 11. Overall performance evaluation of ensemble classifiers for the holdout method.

\begin{tabular}{cccc}
\hline \multicolumn{4}{c}{ Overall Accuracy Rate } \\
\hline Ensemble Method & Holdout & & \\
\hline SVM & RF & $p$-Value \\
\hline Adabging & $98.54 \%$ & $97.18 \%$ & 0.028 \\
Rotation forest & $98.43 \%$ & $98.07 \%$ & 0.686 \\
END & $98.07 \%$ & $98.03 \%$ & 0.893 \\
Random subspace & $98.61 \%$ & $98.03 \%$ & 0.028 \\
\hline
\end{tabular}

Table 12. Overall performance evaluation of ensemble methods for the 10-fold cross-validation.

\begin{tabular}{cccc}
\hline \multicolumn{4}{c}{ Overall Accuracy Rate } \\
\hline \multicolumn{4}{c}{ 10-Fold Cross-Validation } \\
Ensemble Method & SVM & RF & $p$-Value \\
\hline Bagging & $99.07 \%$ & $97.43 \%$ & 0.028 \\
Adaboost & $99.17 \%$ & $98.82 \%$ & 0.028 \\
Rotation forest & $98.43 \%$ & $98.22 \%$ & 0.249 \\
END & $99.20 \%$ & $98.28 \%$ & 0.028 \\
Random subspace & $99.22 \%$ & $97.91 \%$ & 0.028 \\
\hline
\end{tabular}

\subsection{Comparative Analysis}

The comparison of the overall accuracy rate of classification between different methods of classification with previous research work is represented in Table 13.

Table 13. Comparison of overall accuracy of classification with previous research work.

\begin{tabular}{|c|c|c|c|c|}
\hline Reference & $\begin{array}{l}\text { Evaluation } \\
\text { Method }\end{array}$ & Dataset & Classification Method & $\begin{array}{c}\text { Overall } \\
\text { Accuracy Rate }\end{array}$ \\
\hline Proposed classifier & $\begin{array}{c}10 \text {-fold } \\
\text { Cross-validation }\end{array}$ & 10,000 samples & Random subspace-SVM & $99.22 \%$ \\
\hline Ronao and Cho (2017) [21] & $\begin{array}{c}10 \text {-fold } \\
\text { Cross-validation }\end{array}$ & 10,000 samples & $\begin{array}{l}\text { Two stages of continuous } \\
\text { Hidden Markov model }\end{array}$ & $93.18 \%$ \\
\hline Anguita et al. (2013) [25] & Holdout & 10,000 samples & OVA MC-SVM-Gaussian kernel & $96.5 \%$ \\
\hline Kastner et al. (2013) [23] & Holdout & 10,000 samples & $\begin{array}{l}\text { Kernel generalized learning } \\
\text { vector quantization }\end{array}$ & $96.23 \%$ \\
\hline
\end{tabular}

As shown in Table 13, the proposed classifier with Random subspace-SVM achieved an accuracy rate of $99.22 \%$ for 10 -fold cross-validation and $98.74 \%$ for holdout method. These proposed classifiers show improvement of overall accuracy rate of classification on the same dataset (10,000 samples) compared to previous work done by Ranoa and Chao [21] that has achieved an overall accuracy rate of $93.18 \%$, Anguita et al. [25] that obtained overall accuracy rate of 96.5\%, Romero-Paredes et al. [24] that acquired an overall accuracy rate of $96.4 \%$ and Kastner et al. [23] that produced overall accuracy rate of $96.23 \%$. This shows that the Random subspace-SVM ensemble method has the capability to produce higher accuracy due to its ability to find a hyper plane which separates positive and negative training observations, and maximizes the margin between these observations through this hyper plane compared to other methods such as Two stages of continuous Hidden Markov model, OVA MC-SVM-Gaussian kernel, OVO MC-SVM-Linear Kernel majority voting and Kernel generalized learning vector quantization. 
Although RF classifiers have predictive performance comparable to that of the best performing alternatives such as SVMs for classification of HAR, nevertheless, this research shows that SVM has a slight edge over RF. From a small sample size (dataset 1 ) to a larger sample size (dataset 2), the accuracy significantly increased with RF. This is true for SVM as well. This shows that sample size has more impact on the classification accuracy of both the RF and SVM. This is consistent with the results reported by Shao and Lunetta [54], Thanh and Kappas [55], Hasan et al. [56], Solé et al. [57] and Sheshasaayee and Thailambal [58].

It can be established that for the proposed Random subspace-SVM ensemble classification method, the 10-fold Cross-validation evaluation method produced better results than the holdout evaluation method. This is supported by various researchers such as Bengio et al. [59], Kim [60] and Sakr et al. [61].

It can be deduced that the ensemble method gives instinctive, straightforward, well-designed, and strong resolutions for an assortment of machine learning issues. As pointed out by Polikar [16], this method was initially created to enhance classification accuracy by decreasing the modification in classifier outputs. Ensemble methods have since ended up being exceptionally powerful in various areas that are difficult to address utilizing a single model-based classifier. Generally, most ensemble methods are self-determined for the type of base classifier used to construct the ensemble. This is a significant advantage that permits developing a specific kind of classifier that might be known to be most appropriate for a certain application.

\section{Conclusions}

In this study, different ensemble classifiers with different base learner algorithms were implemented to classify six human daily activities based on tri-axial inertial smartphone data. Comparative studies of classification techniques were presented using Bagging, Adaboost, Rotation forest, END and Random subspace with base learner as SVM and RF. The performance measures used to evaluate the classification techniques include overall accuracy, precision, recall, F-measure and ROC. Holdout and 10-fold cross-validation evaluation methods were used in the model evaluation of classification. As seen from the obtained results, Random subspace classifier with SVM gives the best results overall accuracy rate over different ensemble classifiers. The comparison in each activity classification showed the overall results performance of precision, recall, F-measure and ROC and accuracy rate using 10-fold cross-validation method was slightly higher compared to the holdout method. It can be summarized that ensemble classifiers have produced improved performance for the HAR with six different activities such as walking, walking upstairs, walking downstairs, sitting, standing and lying. In future, other methods would be explored to improve the performance.

Author Contributions: I.E. contributed to the conception of the idea and the layout of the research plan, K.N. was involved in the construction of the search queries, participated in the literature search, carried out the research and wrote major sections of the manuscript. L.I.I. and I.E. assisted in reviewing while G.C. edited this manuscript. All authors equally contributed to the rest of the paper. All authors read the manuscript and approved its content.

Funding: Funds for this research was provided by Ministry of Higher Education (MOHE), Malaysia through FRGS Grant, 0153AB-L28, and Universiti Teknologi PETRONAS.

Acknowledgments: The authors would like to thank Universiti Teknologi PETRONAS, Malaysia and HOSEI University, Japan for supporting this work.

Conflicts of Interest: The authors declare no conflict of interest.

\section{References}

1. Abowd, G.D.; Dey, A.K.; Orr, R.; Brotherton, J. Context Tracking in Wearable and Ubiquitous Computing. In Proceedings of the 1st International Symposium on Wearable Computers, Cambridge, MA, USA, 13-14 October 1997; pp. 179-180.

2. Fikri, M.; Fahmi, A.; Negara, P.; Sayeed, S.; Choi, D. Classification Algorithms in Human Activity Recognition Using Smartphones. Int. J. Comput. Inf. Eng. 2012, 6, 77-84. 
3. Wu, W.H.; Bui, A.A.T.; Batalin, M.A.; Au, L.K.; Binney, J.D.; Kaiser, W.J. MEDIC: Medical Embedded Device for Individualized Care. Artif. Intell. Med. 2008, 42, 137-152. [CrossRef] [PubMed]

4. Patel, S.; Park, H.; Bonato, P.; Chan, L.; Rodgers, M. A Review of Wearable Sensors and Systems with Application in Rehabilitation. J. NeuroEng. Rehabil. 2012, 9, 21. [CrossRef] [PubMed]

5. Abowd, G.D.; Atkeson, C.G.; Hong, J.; Long, S.; Kooper, R.; Pinkerton, M. Cyberguide: A Mobile Context Aware Tour Guide. Wirel. Netw. 1997, 3, 421-433. [CrossRef]

6. Alt, F.; Shirazi, A.S.; Pfeiffer, M.; Holleis, P.; Schmidt, A. TaxiMedia: An Interactive Context-Aware Entertainment and Advertising System. In Proceedings of the Informatik 2009: Im Focus das Leben, Beiträge der 39. Jahrestagung der Gesellschaft für Informatik e.V. (GI), Lübeck, Germany, 28 September-2 October 2009.

7. Mitra, S.; Acharya, T. Gesture Recognition: A Survey. IEEE Trans. Syst. Man Cybern. Part C Appl. Rev. 2007, 37, 311-324. [CrossRef]

8. Turaga, P.; Chellappa, R.; Subrahmanian, V.S.; Udrea, O. Machine Recognition of Human Activities: A Survey. IEEE Trans. Circuits Syst. Video Technol. 2008, 18, 1473-1488. [CrossRef]

9. Aggarwal, J.K.; Ryoo, M.S. Human Activity Analysis: A Review. ACM Comput. Surv. 2011, 43, 1-47. [CrossRef]

10. Obermayer, J.L.; Riley, W.T.; Asif, O.; Jean-Mary, J. College Smoking-Cessation Using Cell Phone Text Messaging. J Am. Coll. Health 2004, 53, 71-78. [CrossRef] [PubMed]

11. Aguiar, B.; Silva, J.; Rocha, T.; Carneiro, S.; Sousa, I. Monitoring Physical Activity and Energy Expenditure with Smartphones. In Proceedings of the IEEE-EMBS International Conference on Biomedical and Health Informatics (BHI), Valencia, Spain, 1-4 June 2014; pp. 664-667.

12. Del Rosario, M.B.; Redmond, S.J.; Lovell, N.H. Tracking the Evolution of Smartphone Sensing for Monitoring Human Movement. Sensors 2015, 15, 18901-18933. [CrossRef] [PubMed]

13. Micucci, D.; Mobilio, M.; Napoletano, P. UniMiB SHAR: Dataset for Human Activity Recognition Using Acceleration Data from Smartphones. Appl. Sci. 2017, 7, 1101. [CrossRef]

14. Pires, I.M.; Garcia, N.M.; Pombo, N.; Flórez-Revuelta, F. From Data Acquisition to Data Fusion: A Comprehensive Review and a Roadmap for the Identification of Activities of Daily Living Using Mobile Devices. Sensors 2016, 16, 184. [CrossRef] [PubMed]

15. Jain, A.; Kanhangad, V. Human Activity Classification in Smartphones Using Accelerometer and Gyroscope Sensors. IEEE Sens. J. 2018, 18, 1169-1177. [CrossRef]

16. Polikar, R. Ensemble Based Systems in Decision Making. IEEE Circuits Syst. Mag. 2006, 6, 21-45. [CrossRef]

17. Sagi, O.; Rokach, L. Ensemble Learning: A Survey. Wires Data Min. Knowl. Discovery 2018, 8, e1249. [CrossRef]

18. Anguita, D.; Ghio, A.; Oneto, L.; Parra, X.; Reyes-Ortiz, J.L. Human Activity Recognition on Smartphones Using a Multiclass Hardware-Friendly Support Vector Machine. In Proceedings of the 4th International Workshop, IWAAL 2012, Vitoria-Gasteiz, Spain, 3-5 December 2012.

19. Saha, J.; Chowdhury, C.; Chowdhury, I.R.; Biswas, S.; Aslam, N. An Ensemble of Condition Based Classifiers for Device Independent Detailed Human Activity Recognition Using Smartphones. Information 2018, 9, 94. [CrossRef]

20. Mohamed, R.; Zainudin, M.N.S.; Sulaiman, M.N.; Perumal, T.; Mustapha, N. Multi-Label Classification for Physical Activity Recognition from Various Accelerometer Sensor Positions. J. Inf. Commun. Technol. 2018, 18, 209-231.

21. Ronao, C.A.; Cho, S.-B. Recognizing Human Activities from Smartphone Sensors Using Hierarchical Continuous Hidden Markov Models. Int. J. Distrib. Sens. Netw. 2017, 13, 1-16. [CrossRef]

22. Gao, L.; Bourke, A.K.; Nelson, J. Evaluation of Accelerometer Based Multi-Sensor versus Single-Sensor Activity Recognition Systems. Med. Eng. Phys. 2014, 36, 779-785. [CrossRef] [PubMed]

23. Kastner, M.; Strickert, M.; Villmann, T. A Sparse Kernelized Matrix Learning Vector Quantization Model for Human Activity Recognition. In Proceedings of the Europearn Symposium on Artificial Neural Networks, Computational Intelligence and Machine Learning (ESANN 2013), Bruges, Belgium, 24-26 April 2013.

24. Romera-Paredes, B.; Aung, M.S.H.; Bianchi-Berthouze, N. A One-vs-One Classifier Ensemble with Majority Voting for Activity Recognition. In Proceedings of the Europearn Symposium on Artificial Neural Networks, Computational Intelligence and Machine Learning (ESANN 2013), Bruges, Belgium, 24-26 April 2013.

25. Anguita, D.; Ghio, A.; Oneto, L.; Parra, X.; Reyes-Ortiz, J.L. A Public Domain Dataset for Human Activity Recognition Using Smartphones. In Proceedings of the Europearn Symposium on Artificial Neural Networks, Computational Intelligence and Machine Learning (ESANN 2013), Bruges, Belgium, 24-26 April 2013. 
26. Azmi, M.S.M.; Sulaiman, M.N. Accelerator-Based Human Activity Recognition Using Voting Technique with NBTree and MLP Classifiers. Int. J. Adv. Sci. Eng. Inf. Technol. 2017, 7, 146-152. [CrossRef]

27. Daghistani, T.; Alshammari, R. Improving Accelerometer-Based Activity Recognition by Using Ensemble of Classifiers. (IJACSA) Int. J. Adv. Comput. Sci. Appl. 2016, 7, 128-133. [CrossRef]

28. Catal, C.; Tufekci, S.; Pirmit, E.; Kocabag, G. On the Use of Ensemble of Classifiers for Accelerometer-Based Activity Recognition. Appl. Soft Comput. 2015, 37, 1018-1022. [CrossRef]

29. Gupta, S.; Kumar, A. Human Activity Recognition through Smartphone's Tri-Axial Accelerometer Using Time Domain Wave Analysis and Machine Learning. Int. J. Compt. Appl. 2015, 127, 22-26. [CrossRef]

30. Bayat, A.; Pomplun, M.; Tran, D.A. A Study on Human Activity Recognition Using Accelerometer Data from Smartphones. Procedia Comput. Sci. 2014, 34, 450-457. [CrossRef]

31. Ha, E.T.; Ryu, K.R. Activity Recognition by Smartphone Accelerometer Data Using Ensemble Learning Methods. Int. J. Electr. Comput. Eng. 2014, 8, 480-483.

32. Jiang, W.; Yin, Z. Human Activity Recognition Using Wearable Sensors by Deep Convolutional Neural Networks. In Proceedings of the 23rd ACM international conference on Multimedia, Brisbane, Australia, 26-30 October 2015; pp. 1307-1310.

33. Erdaş, B.C.; Atasoy, I.; Açici, K.; Oğul, H. Integrating Features for Accelerometer-Based Activity Recognition. Procedia Comput. Sci. 2016, 98, 522-527. [CrossRef]

34. Lara, Ó.D.; Prez, A.J.; Labrador, M.A.; Posada, J.D. Centinela: A Human Activity Recognition System Based on Acceleration and Vital Sign Data. Pervasive Mob. Comput. 2012, 8, 717-729. [CrossRef]

35. Pärkkä, J.; Ermes, M.; Korpipää, P.; Mäntyjärvi, J.; Peltola, J.; Korhonen, I. Activity Classification Using Realistic Data from Wearable Sensors. IEEE Trans. Inf. Technol. Biomed. 2006, 10, 119-128. [CrossRef] [PubMed]

36. Maurer, U.; Smailagic, A.; Siewiorek, D.P.; Deisher, M. Activity Recognition and Monitoring Using Multiple Sensors on Different Body Positions. In Proceedings of the International Workshop on Wearable and Implantable Body Sensor Networks (BSN'06), Cambridge, MA, USA, 3-5 April 2006.

37. Reddy, S.; Mun, M.; Burke, J.; Estrin, D.; Hansen, M.; Srivastava, M. Using Mobile Phones to Determine Transportation Modes. ACM Trans. Sens. Netw. 2010, 6, 1-27. [CrossRef]

38. Shoaib, M.; Bosch, S.; Incel, O.D.; Scholten, H.; Havinga, P.J.M. Complex Human Activity Recognition Using Smartphone and Wrist-Worn Motion Sensors. Sensors 2016, 16, 426. [CrossRef] [PubMed]

39. Ali, H.M.; Muslim, A.M. Human Activity Recognition Using Smartphone and Smartwatch. Int. J. Comput. Eng. Res. Trends 2016, 3, 568-576. [CrossRef]

40. Bajrami, G.; Derawi, M.O.; Bours, P. Towards an Automatic Gait Recognition System Using Activity Recognition (Wearable Based). In Proceedings of the 2011 Third International Workshop on Security and Communication Networks (IWSCN), Gjovik, Norway, 18-20 May 2011.

41. Lara, O.D.; Labrador, M.A. A Survey on Human Activity Recognition Using Wearable Sensors. IEEE Commun. Surv. Tutor. 2012, 15, 1192-1209. [CrossRef]

42. Nurhanim, K.; Elamvazuthi, I.; Izhar, L.I.; Ganesan, T. Classification of Human Activity based on Smartphone Inertial Sensor using Support Vector Machine. In Proceedings of the 2017 IEEE 3rd International Symposium in Robotics and Manufacturing Automation (ROMA), Kuala Lumpur, Malaysia, 19-21 September 2017.

43. Rokach, L. Ensemble-Based Classifiers. Artif. Intell. Rev. 2010, 33, 1-39. [CrossRef]

44. Breiman, L. Bagging Predictors. Mach. Learn. 1996, 24, 123-140. [CrossRef]

45. Schapire, R.E.; Singer, Y. Improved Boosting Algorithms Using Confidence-Rated Predictions. Mach. Learn. 1999, 37, 297-336. [CrossRef]

46. Freund, Y.; Schapire, R.E. A Desicion-Theoretic Generalization of on-Line Learning and an Application to Boosting. J. Comput. Syst. Sci. 1995, 55, 119-139. [CrossRef]

47. Rodríguez, J.J.; Kuncheva, L.I.; Alonso, C.J. Rotation Forest: A New Classifier Ensemble Method. IEEE Trans. Pattern Anal. Mach. Intell. 2006, 28, 1619-1630. [CrossRef] [PubMed]

48. Frank, E.; Kramer, S. Ensembles of Nested Dichotomies for Multi-Class Problems. In Proceedings of the twenty-first international conference on Machine learning, Banff, AB, Canada, 4-8 July 2004.

49. Ho, T.K. The Random Subspace Method for Constructing Decision Forests. IEEE Trans. Pattern Anal. Mach. Intell. 1998, 20, 832-844.

50. Amit, Y.; Geman, D. Randomized Inquiries about Shape: An Application to Handwritten Digit Recognition. Available online: http:/ / www.dtic.mil/dtic/tr/fulltext/u2/a290812.pdf (accessed on 27 May 2018). 
51. Hsu, C.-W.; Chang, C.-C.; Lin, C.-J. A Practical Guide to Support Vector Classification. BJU Int. 2008, 101, 1396-1400.

52. Hall, M.; Frank, E.; Holmes, G.; Pfahringer, B.; Reutemann, P.; Witten, I.H. The WEKA Data Mining Software: An Update. ACM SIGKDD Explor. Newslett. 2009, 11, 10-18. [CrossRef]

53. IBM SPSS Software Ver. 20. Available online: http://www.ibm.com (accessed on 24 June 2014).

54. Shao, Y.; Lunetta, R.S. Comparison of support vector machine, neural network, and CART algorithms for the land-cover classification using limited training data points. ISPRS J. Photogramm. Remote Sens. 2012, 70, 78-87. [CrossRef]

55. Thanh Noi, P.; Kappas, M. Comparison of Random Forest, k-Nearest Neighbor, and Support Vector Machine Classifiers for Land Cover Classification Using Sentinel-2 Imagery. Sensors 2018, 18, 18. [CrossRef] [PubMed]

56. Hasan, M.A.M.; Nasser, M.; Pal, B.; Ahmad, S. Support Vector Machine and Random Forest Modeling for Intrusion Detection System (IDS). J. Intell. Learn. Syst. Appl. 2014, 6, 45-52. [CrossRef]

57. Solé, X.; Ramisa, A.; Torras, C. Evaluation of Random Forests on Large-Scale Classification Problems Using a Bag-of-Visual-Words Representation. In Artificial Intelligence Research and Development: Recent Advances and Development; Museros, L., Pujol, O., Agell, N., Eds.; IOS Press: Amsterdam, The Netherlands, 2014; pp. 273-276.

58. Sheshasaayee, A.; Thailambal, G. Comparison of Classification Algorithms in Text Mining. Int. J. Pure Appl. Math. 2017, 116, 425-433.

59. Bengio, Y.; Grandvalet, Y. No unbiased estimator of the variance of k-fold cross-validation. J. Mach. Learn. Res. 2004, 5, 1089-1105.

60. Kim, J.H. Estimating classification error rate: Repeated cross-validation, repeated hold-out and bootstrap. Comput. Stat. Data Anal. 2009, 53, 3735-3745. [CrossRef]

61. Sakr, S.; Elshawi, R.; Ahmed, A.; Qureshi, W.T.; Brawner, C.; Keteyian, S.; Blaha, M.J.; AI-Mallah, M.H. Using Machine Learning on Cardiorespiratory Fitness Data for Predicting Hypertension: The Henry Ford ExercIse Testing (FIT) Project. PLoS ONE 2018, 13, e0195344. [CrossRef] [PubMed]

(C) 2018 by the authors. Licensee MDPI, Basel, Switzerland. This article is an open access article distributed under the terms and conditions of the Creative Commons Attribution (CC BY) license (http:/ / creativecommons.org/licenses/by/4.0/). 